\title{
CHARACTERISTIC CLASSES FOR MODULES OVER GROUPS. I
}

\author{
BY

\section{S. ChARLAP AND A. T. VASQUEZ( $\left.{ }^{1}\right)$}

Suppose $\Phi$ is a group and $M$ is a module over the integral group ring of $\Phi$. Then the homology groups, $H_{i}(M)$, are also $\Phi$-modules. The usual method of seeing this is to use the standard resolution, $S_{*}$, of $Z$ for $M$ because the summands, $S_{i}$, are $\Phi$-modules themselves, and the boundary map is a $\Phi$-homomorphism. However, this complex is a very cumbersome one, and one would like to see if the action of $\Phi$ on $H_{*}(M)$ can be obtained from an arbitrary resolution.

Let $D_{*}$ be any resolution of $Z$ for $M$. The obvious question to ask is whether one can make $D_{i}$ into a $\Phi$-module in any natural way. One of the results of this paper is to show that this can not be done in general. It is the obstructions to this which give rise to the characteristic classes of the title.

The first section develops the notion of a $\Phi$-system for $D_{*}$ which is an approximation to an action of $\Phi$ on $D_{i}$. One part of a $\Phi$-system is, for each $i$ and $\sigma \in \Phi$, a $Z$-homomorphism $A_{i}(\sigma): D_{i} \rightarrow D_{i}$ which is a chain map and satisfies an appropriate semilinearity condition. The point is that $A_{i}(\sigma) \circ A_{i}(\tau) \neq A_{i}(\sigma \tau)$ in general. It is easily seen, however, that they are chain homotopic, and it is such a chain homotopy $U_{i}(\sigma, \tau)$ between $A_{i}(\sigma) \circ A_{i}(\tau)$ and $A_{i}(\sigma \tau)$ which is the other part of a $\Phi$-system and measures the obstruction to the existence of an action of $\Phi$ on $D_{i}$.

If the complex $D_{*}$ is reasonable, these obstructions can be described as follows: For each $\sigma, \tau \in \Phi, U_{i}(\sigma, \tau) \in \operatorname{Hom}\left(D_{i}, D_{i+1}\right)$ defines an element in

$$
\operatorname{Hom}\left(H_{i}(M), H_{i+1}(M)\right)
$$

which is $H^{i}\left(M, H_{i+1}(M)\right)$ if the final (unwritten) coefficients are nice enough. This can be thought of as defining a nonhomogeneous 2-cochain $\omega^{i+1}$ for $\Phi$ with coefficients in $H^{i}\left(M, H_{i+1}(M)\right), \omega^{i+1}$ turns out to be a cocycle and its cohomology class

$$
v^{i+1}(M) \in H^{2}\left(\Phi, H^{i}\left(M, H_{i+1}(M)\right)\right)
$$

is what we call the $i$ th characteristic class of $M . v^{i}(M)$ depends only on $\Phi, M$, and the action of $\Phi$ on $M$.

These algebraic characteristic classes satisfy a naturality condition similar to the one satisfied by topological (e.g. Stiefel-Whitney) ones, and if $M$ is $Z$-free, there is an analogue to the Whitney sum theorem.

Received by the editors January 31, 1967.

$\left.{ }^{1}\right)$ This work was supported in part by NSFGP 4252 and NSFGP 4598-6085. 
Although the original approach to characteristic classes in topology was via obstructions, another method is to use the spectral sequence of a fibration. The algebraic characteristic classes admit a similar interpretation. In $\$ 3$ we show that if $E_{r}^{p, q}$ is the Hochschild-Serre spectral sequence for the split extension of $M$ by $\Phi$, then the map $d_{2}^{p . q}$ is simply obtained by forming the cup product with the class $v^{r}(M)$. In [3] we have shown that $d_{2}$ for an arbitrary extension can be computed by adding the cohomology class of the extension to $v^{p}(M)$, and then taking cup products.

A good part of the paper is devoted to computing characteristic classes in particular cases. We exhibit some cases for which these classes are nonzero, including cases in which $M$ is $Z$-free.

Our main general theorems are that if $M$ is $Z$-free, then $2 v^{i}(M)=0$ and if $v^{2}(M)=0$, then $v^{i}(M)=0$ for all $i$.

In later papers we hope to examine the case where $M$ is finite and to investigate higher order characteristic classes.

\section{Part I. The General Case}

1. Our starting point is a pair of groups $\Phi$ and $M$ and a homomorphism $\phi: \Phi \rightarrow$ the group of automorphisms of $M$. For notational convenience, as is customary, we will suppress $\phi$, denoting $\phi(\sigma)(m)$ by the simpler $\sigma \cdot m$.

Let $R$ denote the group ring, $Z[M]$, of $M$. Let $\left(D_{*}, \partial_{*}\right)$ be a projective resolution of the trivial $M$-module $Z$.

$$
\longrightarrow D_{n} \stackrel{\partial_{n}}{\longrightarrow} D_{n-1} \longrightarrow \cdots \longrightarrow D_{0} \stackrel{\varepsilon}{\longrightarrow} Z \longrightarrow 0
$$

Recall that if $\Gamma$ is an $R$-module, then $H^{n}\left(\operatorname{Hom}_{R}\left(D_{*}, \Gamma\right)\right)$ is denoted by $H^{n}(M, R)$.

Definition. A $\Phi$-system for $\left(D_{*}, \partial_{*}\right)$ consists of two sequences of functions as follows:

$$
A_{n}: \Phi \rightarrow \operatorname{Hom}_{z}\left(D_{n}, D_{n}\right) \text { and } U_{n}: \Phi \times \Phi \rightarrow \operatorname{Hom}_{z}\left(D_{n}, D_{n+1}\right)
$$

subject to the conditions listed below:

(i) $\partial_{n} A_{n}(\sigma)=A_{n-1}(\sigma) \partial_{n}, \quad n \geqq 1$,

(ii) $\varepsilon A_{0}(\sigma)=\varepsilon$,

(iii) " $A_{n}(\sigma)$ is $\sigma$-linear", i.e.

$$
A_{n}(\sigma)(r \cdot d)=\sigma(r) \cdot A_{n}(\sigma)(d) \text { for } d \in D_{n} \text { and } r \in R=Z[M],
$$

(i') $\partial_{n+1} U_{n}(\sigma, \tau)+U_{n+1}(\sigma, \tau) \partial_{n}=A_{n}(\sigma \tau)-A_{n}(\sigma) \circ A_{n}(\tau), \quad n \geqq 1$,

(ii') $\partial_{1} U_{0}(\sigma, \tau)=A_{0}(\sigma \tau)-A_{0}(\sigma) \circ A_{0}(\tau)$,

(iii') $U_{n}(\sigma, \tau)$ is $\sigma \tau$-linear.

RemarKS. 1. $A_{n}$ is not a homomorphism, i.e. $A_{n}(\sigma \tau) \neq A_{n}(\sigma)+A_{n}(\tau)$.

2. Intuitively we would like to think of $A_{n}$ as defining an action of $\Phi$ on $D_{*}$. This is not what happens in general, i.e. $A_{n}(\sigma \tau) \neq A_{n}(\sigma) \circ A_{n}(\tau)$. 
3. The homomorphisms $A_{n}(\sigma) \in \operatorname{Hom}_{z}\left(D_{n}, D_{n}\right)$ are $Z$-homomorphisms and not $R$-homomorphisms. Rather they satisfy the semilinear condition (iii). Similarly for $U_{n}$.

EXAMPLE 1. Let $\left(S_{*}, \partial_{*}\right)$ be the standard resolution, i.e. $S_{n}=$ the free abelian group generated by the set $M \times \cdots \times M(n+1$ times). Then a $\Phi$-system con be defined by the equations

$$
A_{n}(\sigma)\left(m_{0}, \ldots, m_{n}\right)=\left(\sigma\left(m_{0}\right), \ldots, \sigma\left(m_{n}\right)\right) .
$$

In this case, of course, $A_{n}(\sigma \tau)=A_{n}(\sigma) A_{n}(\tau)$ so we can take $U_{n} \equiv 0$. We should remark that in many cases the $U_{n}$ 's are more significant than the $A_{n}$ 's. One can think of the $U_{n}$ 's as representing the obstruction to finding an action of $\Phi$ on $D_{*}$.

Note that if $D_{n}^{\sigma}$ denotes $D_{n}$ with a new $R$ module structure given by $m \circ x_{n}$ $=\sigma(m) x_{n}$, then $A_{n}(\sigma) \in \operatorname{Hom}_{R}\left(D_{n}, D_{n}^{\sigma}\right)$. This observation reduces the proof of the following proposition to the standard ones.

Proposition 1. $\Phi$-systems exist for any such $\left(D_{*}, \partial_{*}\right)$.

EXAMPLE 2. Let $Z_{r}$ act on $Z_{s}$ by $\sigma \cdot t=t^{q}$ where $q^{r}=1$ modulo $s$. Here $\sigma$ and $t$ are generators of multiplicative groups $Z_{r}$ and $Z_{s}$ of orders $r$ and $s$ respectively.

Let $\Delta=1-t \in R=Z\left[Z_{s}\right], N=1+t+\cdots+t^{s-1} \in R$, and $\alpha=1+t+\cdots+t^{q-1} \in R$. Then it is well known that there is a resolution of $Z$ as follows:

$$
\longrightarrow D_{1} \stackrel{\partial_{1}}{\longrightarrow} D_{0} \stackrel{\varepsilon}{\longrightarrow} Z \longrightarrow 0
$$

where $D_{i}=R, \varepsilon(t)=1, \partial_{2 k+1}(1)=\Delta$ and $\partial_{2 k}(1)=N$. It is trivial to verify that $A_{n}(\sigma)$ may be chosen such that

$$
A_{2 k}(\sigma)(1)=\alpha^{k}, \quad A_{2 k+1}(\sigma)(1)=\alpha^{k+1} .
$$

Define $A_{n}\left(\sigma^{i}\right)=i$-fold iteration of $A_{n}(\sigma)$ for $0 \leqq i<r$. It is convenient to let $p=q^{r-1} / s$.

Lemma. Let $U_{n} \in \operatorname{Hom}_{R}\left(D_{n}, D_{n+1}\right)$ be defined by

$$
\begin{aligned}
U_{2 k} & =0 \\
U_{2 k+1}(1) & =\frac{\left(q^{r}\right)^{k+1}-1}{s} \in R .
\end{aligned}
$$

Then $\partial_{n+1} U_{n}+U_{n-1} \partial_{n}=A_{n}(\sigma)^{r}-$ identity $(n \geqq 1)$ and $\partial_{1} U_{0}=0$.

Proof. This reduces to establishing

$$
\frac{\left(q^{r}\right)^{k}-1}{s} N=\left(A_{2 k}(\sigma)-\mathrm{id}\right)(1)
$$

Now $A_{2 k}(\sigma)^{2}(1)=A_{2 k}(\sigma)\left(\alpha^{k}\right)=A_{2 k}(\sigma)\left(\alpha^{k} \cdot 1\right)=\sigma\left(\alpha^{k}\right) A_{2 k}(\sigma)(1)=\sigma\left(\alpha^{k}\right) a^{k}$, etc. Thus $\left(A_{2 k}(\sigma)^{r}-\mathrm{id}\right)(1)=\left\{\sigma^{r-1}(\alpha) \cdots \sigma(\alpha) \alpha\right\}^{k}-1$; thus we must show that

$$
\beta^{k}-1=\left(\left(q^{r}\right)^{k}-1 \cdot N\right) / s
$$


where $\beta=\sigma^{r-1}(\alpha) \cdots \sigma(a) \cdot \alpha \in R$. But $\partial_{1} \beta=\partial_{1} A_{1}(\sigma)^{r}(1)=A_{0}(\sigma)^{r} \partial_{1}(1)=\partial_{1}(1)$, i.e. $\Delta \beta=\Delta$. So $\Delta \beta^{k}=\Delta$. Thus $\Delta\left(\beta^{k}-1\right)=0$, or $\beta^{k}-1 \in \operatorname{Ker} \partial_{1}=\operatorname{Im} \partial_{2}=\{n N \mid n \in Z\}$. But then $\beta^{k}-1=n_{k} N$ for some integer $n_{k}$. Applying the ring homomorphism $\varepsilon: R \rightarrow Z$ we conclude that

$$
\varepsilon(\beta)^{k}-\varepsilon(1)=n_{k} \in(N) \text { or }\left(q^{r}\right)^{k}-1=n_{k} \cdot s .
$$

This completes the proof of the lemma.

It is easy now to see how to choose $U_{n}\left(\sigma^{i}, \sigma^{j}\right)$. In fact we may choose

$$
\begin{aligned}
U_{n}\left(\sigma^{i}, \sigma^{j}\right) & =0 & & \text { if } i+j<r \\
& =A_{n}\left(\sigma^{i+j}\right) \circ U_{n}(1) & & \text { if } i+j \geqq r .
\end{aligned}
$$

Returning to the general theory we suppose that $\Gamma$ is both an $M$-module and a $\Phi$-module such that

$$
\sigma(m \cdot \gamma)=\sigma(m) \cdot \sigma(\gamma) \quad \forall \sigma \in \Phi, m \in M \text { and } \gamma \in \Gamma .
$$

Then we can extend $A_{*}$ to $\operatorname{Hom}_{R}\left(D_{*}, \Gamma\right)$ by $\left(A_{n}(\sigma) \cdot f\right)\left(x_{n}\right)=\sigma\left(f\left(A_{n}\left(\sigma^{-1}\right) x_{n}\right)\right)$. The following is readily verified.

Proposition 2. The above map induces an action of $\Phi$ on $H^{n}(M ; \Gamma)$. This action is independent of the $\Phi$-system and of the particular resolution $\left(D_{*}, \partial_{*}\right)$.

Of course there is entirely analogously an action of $\Phi$ on $H_{n}(M, \Gamma)$ (i.e. on the homology of $\left.D_{*} \otimes_{R} \Gamma\right)$. We will denote by $\sigma_{*}(\chi)$ this action of $\sigma$ on $\chi \in H_{n}(M, \Gamma)$.

This action is usually defined via the complex $\left(S_{*}, \partial_{*}\right)$ of Example 1 (see e.g. [4]).

2. Characteristic classes. Let $k$ be a principal ideal domain on which $M$ acts trivially and assume further that $H_{n}(M, k)$ is $k$-projective. (It seems likely that this assumption is unduly restrictive but it is convenient.) Thus from the universal coefficient sequence we know that $H^{n}(M, A) \simeq \operatorname{Hom}_{k}\left(H_{n}(M, k), A\right)$ where $A$ is a $k$-module on which $M$ acts trivially.

We remark that these assumptions are automatically satisfied in two important cases:

(a) $k$ is a field,

(b) $M$ is a finitely generated free abelian group and $k=Z$.

We will omit specific references to $k$ in much of what follows.

Let $f^{n} \in \operatorname{Hom}_{R}\left(D_{n}, H_{n}(M, k)\right)$ be a cocycle representing the cohomology class corresponding to the identity map in $\operatorname{Hom}_{k}\left(H_{n}(M, k), H_{n}(M, k)\right)$. It is clear that $A_{n}(\sigma) \cdot f^{n}$ represents the same cohomology class, so for each $\sigma \in \Phi$, there is some $F_{\sigma}^{n-1} \in \operatorname{Hom}_{R}\left(D_{n-1}, H_{n}(M)\right)$, such that $A_{n}(\sigma) \cdot f^{n}-f^{n}=F_{\sigma}^{n-1} \partial_{n}$. Define $u^{n}(\sigma, \tau)$ $\in \operatorname{Hom}_{R}\left(D_{n-1}, H_{n}(M)\right)$ by

$$
u^{n}(\sigma, \tau)=A_{n-1}(\sigma) \cdot F_{\imath}^{n-1}-F_{\sigma \tau}^{n-1}+F_{\sigma}^{n-1}+(\sigma, \tau)_{*}\left[f^{n} U_{n-1}\left(\tau^{-1}, \sigma^{-1}\right)\right] .
$$


Lemma. $u^{n}(\sigma, \tau)$ is a cocycle and hence defines an element $\omega^{n}(\sigma, \tau)$, of $H^{n-1}\left(M, H_{n}(M, k)\right)$.

\section{Proof.}

$$
\begin{aligned}
\delta^{n-1} u^{n}(\sigma, \tau)= & u^{n}(\sigma, \tau) \partial_{n} \\
= & \sigma_{*} \cdot F_{\tau}^{n-1} A_{n-1}\left(\sigma^{-1}\right) \partial_{n}+F_{\sigma \tau}^{n-1} \partial_{n}+F_{\sigma}^{n-1} \circ \partial_{n} \\
& +(\sigma \tau)_{*} f^{n}\left(-\partial_{n} U_{n}\left(\tau^{-1}, \sigma^{-1}\right)-A_{n}\left(\tau^{-1}\right) A_{n}\left(\sigma^{-1}\right)+A_{n}\left(\tau^{-1} \sigma^{-1}\right)\right) \\
= & \sigma_{*}\left(A_{n}(\tau) \cdot f^{n}-f^{n}\right) A_{n}\left(\sigma^{-1}\right)-F_{\sigma \tau}^{n-1} \partial_{n}+F_{\sigma}^{n-1} \partial_{n} \\
& +\left\{-(\sigma \tau)_{*} f^{n} A_{n}\left(\tau^{-1}\right) A_{n}\left(\sigma^{-1}\right)+A_{n}(\sigma \tau) \cdot f^{n}\right\} \\
= & \sigma_{*} \tau_{*} f^{n} A_{n}\left(\tau^{-1}\right) A_{n}\left(\sigma^{-1}\right)-A_{n}(\sigma) \cdot f^{n}-\left\{A_{n}(\sigma \tau) \cdot f^{n}-f^{n}\right\} \\
& +\left\{A_{n}(\sigma) \cdot f^{n}-f^{n}\right\}+\left\{-(\sigma \tau)_{*} f^{n} A_{n}\left(\tau^{-1}\right) A_{n}\left(\sigma^{-1}\right)+A_{n}(\sigma \tau) \cdot f^{n}\right\} \\
= & 0 .
\end{aligned}
$$

THEOREM 1. The function $\omega^{n}: \Phi \times \Phi \rightarrow H^{n-1}\left(M, H_{n}(M, k)\right)$ is a $\Phi$-cocycle and represents therefore an element $v^{n} \in H^{2}\left(\Phi, H^{n-1}\left(M, H_{n}(M, k)\right)\right)$.

Proof. $\left(\delta \omega^{n}\right)(\sigma, \tau, \rho)=A_{n}(\sigma) \sigma \cdot \omega^{n}(\tau, \rho)-\omega^{n}(\sigma \tau, \rho)+\omega^{n}(\sigma, \tau \rho)-\omega^{n}(\sigma, \tau)$. We work of course with a representative cocycle. Such a one is obtained by replacing $\omega$ by $u$.

After expanding and cancelling many pairs of terms we obtain the expression

$$
\begin{aligned}
& \sigma \tau F_{\rho}^{n-1}\left(A_{n-1}\left(\tau^{-1}\right) A_{n-1}\left(\sigma^{-1}\right)-A_{n-1}\left(\tau^{-1} \sigma^{-1}\right)\right) \\
& +\sigma \tau \rho f^{n}\left(U_{n-1}\left(\rho^{-1}, \tau^{-1}\right) A_{n-1}\left(\sigma^{-1}\right)-U_{n-1}\left(\rho^{-1}, \tau^{-1} \sigma^{-1}\right)\right. \\
& \left.\quad+U_{n-1}\left(\rho^{-1} \tau^{-1}, \sigma^{-1}\right)-\sigma \tau f^{n} U_{n-1}\left(\tau^{-1}, \sigma^{-1}\right)\right) .
\end{aligned}
$$

Using property ( $\left.i^{\prime}\right)$ of $\Phi$-systems the first term becomes

$$
\begin{aligned}
-\sigma \tau F_{\rho}^{n-1}\left(\partial_{n} U_{n-1}\left(\tau^{-1}, \sigma^{-1}\right)\right. & \left.+U_{n-2}\left(\tau^{-1}, \sigma^{-1}\right) \partial_{n-1}\right) \\
= & -\sigma \tau\left\{\rho f^{n} A_{n}\left(\rho^{-1}\right)-f^{n}\right\} U_{n-1}\left(\tau^{-1}, \sigma^{-1}\right)+\text { a coboundary. }
\end{aligned}
$$

Thus except for a coboundary the whole expression becomes, except for sign,

$$
\begin{aligned}
+\sigma \tau \rho f^{n}\left\{A_{n}\left(\rho^{-1}\right) U_{n-1}\left(\tau^{-1}, \sigma^{-1}\right)-U_{n-1}\right. & \left(\rho^{-1}, \tau^{-1}\right) A_{n-1}\left(\sigma^{-1}\right) \\
& \left.+U_{n-1}\left(\rho^{-1}, \tau^{-1} \sigma^{-1}\right)-U_{n-1}\left(\rho^{-1} \tau^{-1}, \sigma^{-1}\right)\right\} .
\end{aligned}
$$

If we rewrite this as $\sigma \tau \rho \omega^{n-1}(\sigma, \tau \rho)$, a direct computation shows that

$$
\partial_{n}, \omega^{n-1}+\omega^{n-2} \partial_{n-1}=0 \text { and } \partial_{1} \omega^{0}=0
$$

Since $D_{*}$ is a projective resolution it follows that for some $(\sigma \tau \rho)^{-1}$-linear functions $B_{n} \in \operatorname{Hom}\left(D_{n}, D_{n+2}\right)$ we have $\partial_{n+2} B_{n}+B_{n-1} \partial_{n}=\omega^{n}$ and $\partial_{z} B_{0}=\omega^{0}$. Taking account of the fact that $f^{n}$ is a cocycle, this shows that the whole expression is a coboundary and thus $\left(\delta \omega^{n}\right)(\sigma, \tau, \rho)=0 \in H^{n-1}\left(M, H_{n}(M, k)\right)$ which was to be proved.

THEOREM 2. The cohomology class of $\omega^{n}$ depends only on the homomorphism $\phi: \Phi \rightarrow$ Aut $(M)$. More particularly, it is independent of the choice of $f^{n}, F_{\sigma}^{n-1}$, the $\Phi$-system and the particular resolution $D_{*}$. 
We omit the proof since it is even more tedious than the previous ones, and no more difficult. We suggest however that the effect of the various choices be considered one-by-one.

Definition. The cohomology class of $\omega^{n}$ in $H^{2}\left(\Phi, H^{n-1}\left(M, H_{n}(M, k)\right)\right)$ is called the $n$th characteristic class. It is denoted $v^{n}(M)$ or sometimes simply $v^{n}$.

Naturality. Let $h: \Phi^{\prime} \rightarrow \Phi$ be any homomorphism. Then $\Phi^{\prime}$ acts on $M$ via $h$ and the action of $\Phi$ on $M$. For notational convenience we denote by $M^{\prime}, M$ with this action of $\Phi^{\prime}$. Thus $v^{n}\left(M^{\prime}\right) \in H^{2}\left(\Phi^{\prime}, H^{n-1}\left(M^{\prime}, H_{n}\left(M^{\prime}, k\right)\right)\right)$ are defined. It is trivial to see that $h$ induces a homomorphism.

$$
h^{*}: H^{2}\left(\Phi, H^{n-1}\left(M, H_{n}(M)\right)\right) \rightarrow H^{2}\left(\Phi^{\prime}, H^{n-1}\left(M^{\prime}, H_{n}\left(M^{\prime}\right)\right)\right)
$$

and to prove the following proposition.

TheOREM 3 (NATURALITY). $h^{*}\left(v^{n}(M)\right)=v^{n}\left(M^{\prime}\right)$.

Example 2 (Continued). We choose $k=Z_{s}$. Thus $D_{n} \otimes_{R} Z_{s}=R \otimes_{R} Z_{s} \simeq Z_{s}$ and $\partial_{n} \otimes 1=0$. Thus $H_{n}\left(Z_{s}, Z_{s}\right) \cong D_{n} \otimes_{R} Z_{s} \cong Z_{s}$ for $n \geqq 1$. It is clear that $\gamma_{n}=1 \otimes 1$ $\in Z_{n} \otimes Z_{s}$ is a generator and that $\sigma_{*}\left(\gamma_{2 k}\right)=A_{2 k}(\sigma)(1) \otimes 1=\alpha^{k} \otimes 1=\alpha^{k-1} \otimes q$ $=\cdots=1 \otimes q^{k}=q^{k} 1 \otimes 1=q^{k} \gamma_{2 k}$. Similarly $\sigma_{*}\left(\gamma_{2 k+1}\right)=q^{k+1} \gamma_{2 k+1}$. Now $f^{n} \in$ $\operatorname{Hom}_{R}\left(D_{n}, H_{n}\right)$ is characterized by $f^{n}(1)=\gamma_{n}$. By definition $A_{2 k}\left(\sigma^{-1}\right) \cdot f^{2 k}=\sigma_{*}^{-1}$ $\circ f^{2 k} \circ A_{2 k}(\sigma)$ and so its value on 1 is $\sigma_{*}^{-1} f^{2 k}\left(\alpha^{k} \cdot 1\right)=\sigma_{*}^{-1}\left(q^{k} \cdot \gamma_{2 k}\right)=\gamma_{2 k}$; i.e. $A_{2 k}\left(\sigma^{-1}\right)$ $\cdot f^{2 k}=f^{2 k}$. Similarly $A_{n}\left(\sigma^{i}\right) \cdot f^{n}=f^{n}$ so $F_{\sigma}^{n-1}=0$ is legitimate. Thus $u^{n}\left(\sigma^{i}, \sigma^{j}\right)=$ $-\sigma_{*}^{i+j} f^{n} \circ U_{n-1}\left(\sigma^{-i}, \sigma^{-j}\right)$. Thus we conclude that $v^{2 k+1}=0$ (since $U_{2 k}=0$ ). To compute further however we must determine the $\Phi$-module structure of $H^{n-1}\left(M, H_{n}(M)\right)=\operatorname{Hom}\left(H_{n-1}, H_{n}\right)$ in this case. It is easy from the above to see that for even $n, \sigma$ acts trivially while for odd $n, \sigma_{*}$ is multiplication by $q$. The next task is to describe $H^{2}\left(\Phi, H^{k-1}\left(M, H_{n}(M)\right)\right)$. Since $\Phi$ is cyclic in this case we may make use of the fact that $H^{2}(\Phi, A)=A^{\Phi} / \Sigma \cdot A$, i.e. the quotient of the fixed elements by the image of $\Sigma$ where $\Sigma=1+\sigma+\sigma^{2}+\cdots+\sigma^{r-1}$. If $\omega \Phi \times \Phi \rightarrow A$ is a twococycle representing an element $\{\omega\} \in H^{2}(\Phi, A)$ then it is readily verified that $\sum_{i=0}^{r-1} \omega\left(\sigma^{i}, \sigma\right) \in A^{\Phi}$ and represents the corresponding class in $A^{\Phi} / \Sigma A$. Our attention is therefore focused on

$$
\sigma_{*}^{r} f^{2 k} U_{2 k-1}=f^{2 k} U_{2 k-1}=\frac{q^{r k}-1}{s} \cdot f^{2 k}
$$

Putting everything together

$$
v^{2 k} \in H^{2}\left(\Phi, H^{2 k-1}\left(M, H_{2 k}(M)\right)\right)=Z_{s} / r \cdot Z_{s}
$$

and corresponds to $-\left(q^{r k}-1\right) / s$. Now it is easy to see that if $\left(q^{r}-1\right) / s=p$ then $\left(q^{r k}-1\right) / s=k \cdot p \bmod s$.

Summarizing. If $Z_{r}$ acts on $Z_{s}$ by $\sigma(t)=t^{q}$ where $q^{r}-1=p s$ then $v^{2 k+1}=0$ and $v^{2 k} \in h^{2}\left(Z_{r}, H^{2 k-1}\left(Z_{s}, H_{2 k}\left(Z_{s}\right)\right)\right) \simeq Z_{s} / r \cdot Z_{s}$ corresponds to $-k \cdot p$.

In the special case $r=2, s=8, q=5$ we conclude that $v^{4 m+2} \neq 0$ for any $m$ while all others are zero. 
We remark that this shows that one cannot find a $\Phi$-system $A_{n}^{\prime}$ for the complex of the example satisfying $A_{n}^{\prime}(\sigma \tau)=A_{n}^{\prime}(\sigma) \circ A_{n}^{\prime}(\tau)$. For then $U_{n-1}^{\prime}=0$ would be permissible and then $v^{n}=0$ would be a consequence.

3. The second differential. Let $G=$ the semidirect product of $M$ and $\Phi$. Thus $M$ is a normal subgroup of $G$ with quotient $\Phi$. Indeed we can represent each element, $g$, of $G$ uniquely as a pair $(m, \sigma) \in M \times \Phi$. The multiplication in $G$ is then

$$
\left(m_{1}, \sigma_{1}\right)\left(m_{2}, \sigma_{2}\right)=\left(m_{1}+\sigma_{1}\left(m_{2}\right), \sigma_{1} \sigma_{2}\right)
$$

Hochschild and Serre have shown in [4], that if $B$ is some appropriate coefficient group, there is a spectral sequence

$$
E_{r}^{p, q}(B) \Rightarrow H^{n}(G ; B),
$$

where $E_{2}^{p, q}(B)=H^{p}\left(\Phi, H^{q}(M, B)\right)$.

In [2] and [3], the present authors found an interpretation of the second differential in this spectral sequence (actually the emphasis there is the comparison of this spectral sequence with those of other extensions of $\Phi$ by $M$ ). We wish to recall that proposition and to relate the characteristic classes with the differential.

First fix an integer $n$. The universal coefficient theorem gives

$$
H^{n}(M, k) \cong \operatorname{Hom}_{k}\left(H_{n}(M, k), k\right) .
$$

Thus $H^{n}(M, k)$ and $H_{n}(M, k)$ are paired to $k$. This induces a cup-product pairing of spectral sequences as follows:

$$
\bar{E}_{r}^{p, q} \otimes \widetilde{E}_{r}^{p^{\prime} q, q^{\prime}} \rightarrow E_{r}^{p+p^{\prime}, q+q^{\prime}}
$$

where

$$
\begin{aligned}
& \bar{E}_{r}^{p, q}=E_{r}^{p, q}\left(H^{N}(M, k)\right) \\
& \tilde{E}_{r}^{p, q}=E_{r}^{p, q}\left(H_{N}(M, k)\right)
\end{aligned}
$$

and

$$
E_{r}^{p, q}=E_{r}^{p, q}(k) .
$$

In particular $\widetilde{E}_{2}^{0, N} \simeq H^{0}\left(\Phi, \operatorname{Hom}_{k}\left(H_{N}(M, k), H_{N}(M, k)\right)\right)$. This group contains an element $f^{N}$, which corresponds to the identity in $\operatorname{Hom}_{k}\left(H_{N}(M, k), H_{N}(M, k)\right)$. Note also that $\bar{E}_{2}^{p, 0}=H^{p}\left(\Phi, H^{0}\left(M, H^{N}(M, k)\right)\right)$ is isomorphic to $H^{p}\left(\Phi, H^{N}(M, k)\right)$ $=E_{2}^{p, N}$. Calling this isomorphism $\theta$, Proposition 2.2 of [3] can be stated as follows.

Proposition 2. Let $\chi \in E_{2}^{p, N}$ then

$$
d_{2}(\chi)=(-1)^{p} \theta(\chi) \cup d_{2}\left(f^{N}\right) .
$$

We should remark that this is not at all deep; it is confusing due to the superabundance of notation. The significance of it is that it "reduces" the computation of $d_{2}$ to that of $d_{2}\left(f^{N}\right) \in H^{2}\left(\Phi, H^{N-1}\left(M, H_{N}(M, k)\right)\right)$. The following proposition can be interpreted as giving a computational hold on $d_{2}\left(f^{N}\right)$. 
THEOREM 4. $v^{N}(M)=d_{2}\left(f^{N}\right)$.

Proof. The proof of Theorem 6.1 of [3] shows that, using the standard complex $\left(S_{*}, \partial_{*}\right), d_{2}\left(f^{N}\right)$ is represented by the cocycle

$$
Y^{n}(\sigma, \tau)=A_{n-1}(\sigma) \cdot F_{\tau}^{n-1}-F_{\sigma \tau}^{n-1}+F_{\sigma}^{n-1} \in \operatorname{Hom}_{R}\left(S_{N-1}, H_{N}(M)\right) .
$$

For this complex $U_{k}=0$ is permissible. Thus $\left\{Y^{N}\right\}=\left\{u^{N}\right\}$.

We remark that our interest in the classes $v^{N}$ stems from this theorem. One would hope that it would give some information about $H^{n}(G, k)$ (which it does). However, the proposition can be turned around to give us information about $v^{N}$. The proposition below is such a situation.

Proposition 3. $v^{1}(M)=0$.

Proof. Since $d_{2}: E_{2}^{0,1} \rightarrow E_{2}^{2,0}$ is such that $v^{1}(M)=d_{2}\left(f^{1}\right)$ we see that $v^{1} \neq 0$ implies $E_{3}^{2,0} \neq E_{2}^{2,0}$. But $E_{\infty}^{2,0}(B)=E_{2}^{2,0}(B) / d_{2}\left(E_{2}^{0,1}(B)\right)$. Also $E_{2}^{N, 0}(B)=H^{n}\left(\Phi_{0}, H^{0}(M, B)\right)$ $\cong H^{n}(\Phi, B)$. Under this identification indeed

$$
E_{\infty}^{n, 0}(B) \cong \text { Image }\left(H^{n}(\Phi, B) \stackrel{\pi^{*}}{\longrightarrow} H^{n}(G, B)\right)
$$

where $\pi$ is the canonical projection from $G$ to $\Phi=G / M$. Since there is a homomorphism $\rho: \Phi \rightarrow G$ such that $\pi \rho=1_{\Phi}$, it follows that $\pi^{*}$ is a monomorphism and hence that $E_{\infty}^{n, 0}(B)=E_{2}^{n, 0}(B)$. Thus we conclude that $d_{2} E_{2}^{0,1}(B) \rightarrow E_{2}^{2,0}(B)$ is zero. For appropriate choice of $B$ this gives $d_{2}=0$.

\section{Part II. The Free Abelian Case}

1. We assume throughout Part II that $M$ is a free abelian group. We will specialize the preceding by choosing $k=Z$ although that is not strictly necessary for much of what we do.

Our first task is to describe a particular resolution $\left(D_{*}, \partial_{*}\right)$. Let $\left\{m_{i}\right\} i \in J$ be an indexed free basis for $M$. Let $N=\mathrm{a}$ free $(R=Z[M])$ module on symbols $\left\{X_{i}\right\} i \in J$. It is well known that there is an acyclic resolution of $Z$ via $R$-free modules as follows:

(4a) $D_{n}=\Lambda_{R}^{n} N, n \geqq 0$.

(4b) $\varepsilon: D_{0} \equiv R \rightarrow Z$ satisfies $\varepsilon(m)=1 \forall m \in M$.

(4c) $\partial_{1}: D_{1} \rightarrow D_{0}$ satisfies $\partial_{1} \chi_{i}=m_{i}-1 \in R \forall i \in J$.

(4d) $\partial_{m+n}(X \wedge Y)=\partial_{n} X \wedge Y+(-1)^{n} X \wedge \partial_{m} Y$ for $X \in D_{n}$ and $Y \in D_{m}$.

Clearly $\partial \otimes$ id $=0$ and so $H_{n}(M, Z) \cong D_{n} \otimes_{R} Z \cong \Lambda^{n}\left(D_{1} \otimes_{R} Z\right) \cong \Lambda^{n} M$. The multiplication in $D_{*}$ corresponds to Pontrjagin multiplication in $H_{*}(M)$. We will exploit this multiplicative structure below.

THEOREM 5. $2 v^{2}(M)=0$ for free abelian groups $M$.

Proof. Consider $f \in \operatorname{Hom}_{R}\left(S_{2}, H_{2}(M)\right)$ where $f\left(1, m_{1}, m_{2}\right)=m_{1} \wedge m_{2} \in \Lambda^{2} M$ $\cong H_{2}(M)$. The lemma below shows that $f$ and $-2 f^{2}$ are cohomologous, i.e., there 
is an $h \in \operatorname{Hom}_{R}\left(S_{1}, H_{2}\right)$ such that $f=-2 f^{2}+\delta h$. According to equation (2), in this case, $v^{2}$ is given up to sign by $\sigma \cdot F_{\tau}^{1}-F_{\sigma \tau}^{1}+F_{\sigma}^{1}$ where $\delta F_{\sigma}^{1}=\sigma \cdot f^{2}-f^{2}$. Since $\sigma \cdot f=f$ we conclude that $0=-2 \delta F_{\sigma}^{1}+\delta(\sigma \cdot h-h)$. Thus we may define $g_{\sigma} \in H\left(M, H_{2}(M)\right)$ by $g_{\sigma}=$ the cohomology class of $-2 F_{\sigma}^{1}+\sigma \cdot h-h$. Now it is clear that

$$
(\delta g)(\sigma, \tau)=\sigma \cdot g \tau-g \sigma \tau+g \sigma=-2\left\{\sigma \cdot F_{\tau}^{1}-F_{\sigma \tau}^{1}+F_{\sigma}^{1}\right\} .
$$

Thus $2 v^{2}(M)=0$.

LEMMA. $f$ represents the class in $H^{2}\left(M, H_{2}(M)\right) \simeq \operatorname{Hom}\left(H_{2}(M), H_{2}(M)\right)$ corresponding to twice the identity map.

Proof. We construct part of a chain transformation $\phi: D_{*} \rightarrow S_{*}$. Choose $\phi_{0}(1)=(1), \phi_{1}\left(X_{i}\right)=+\left(1, m_{i}\right) \in S_{1}$, and

$$
\phi_{2}\left(X_{i_{1}} \wedge X_{i_{2}}\right)=+\left(1, m_{i_{1}}, m_{i_{2}}\right)-\left(m_{i_{1}}, m_{i_{2}}, m_{i_{1}}, m_{i_{2}}\right)
$$

for $i_{1}<i_{2}$ relative to some ordering of $J$. It is readily verified that $\partial_{2} \phi_{2}=\phi_{1} \partial_{2}$, $\partial_{1} \phi_{1}=\phi_{0} \partial_{1}$, and $\varepsilon \phi_{0}=\varepsilon$. It is also trivial that $f$ is in fact a cocycle. Thus the cohomology class is determined by $f \phi_{2} \varepsilon: \operatorname{Hom}_{R}\left(D_{2}, H_{2}(M)\right)$. Now $\left(f \phi_{2}\right)\left(X_{i_{1}} \wedge X_{i_{2}}\right)$ $=+m_{i_{1}} \wedge m_{i_{2}}-\left(m_{i_{1}}^{-1} m_{i_{2}}\right) \wedge m_{i_{2}}=+2 m_{i_{1}} \wedge m_{i_{2}}$, then $+f \phi_{2}$ represents twice the identity.

We will see later that this implies $2 v^{n}(m)=0$ for all $n$.

For the moment we record the following trivial facts.

Proposition 3. Let $A_{*}, U_{*}$ be any $\Phi$-system for $\left(D_{*}, \partial_{*}\right)$. Then we may choose $F_{\sigma}=0$ for all $\sigma \in I$ and consequently

$$
\omega^{n}(\sigma, \tau)=+\left\{\sigma_{*} \tau_{*} f^{n} U_{n-1}\left(\tau^{-1}, \sigma^{-1}\right)\right\}
$$

is a representative for $v^{n}(M)$. Since $f^{n}: \Lambda_{R}^{n} D_{1} \rightarrow H_{n}(M)$ is $R$-linear and $M$ acts trivially on $H_{n}(M), f^{n}$ actually factors through the canonical projection

$$
\Lambda_{R}^{n} D_{1} \rightarrow \Lambda_{R}^{n} D_{1} \otimes_{R} Z=\Lambda^{n}\left(D_{1} \otimes_{R} Z\right)=\Lambda^{n} M=H_{n}(M) .
$$

So does $A_{n}(\sigma) \cdot f^{n}$ and in fact they both correspond after the canonical projection to the identity map. Hence they are equal, and so $F_{\sigma}=0$ is appropriate.

Proposition 4. There is a $\Phi$-system $\left\{A_{n}\right\}$ for the above complex satisfying

(4e) $A_{0}(\sigma) m=\sigma(m), \quad m \in M \subset R=D_{0}$ and $\sigma \in I$, and

(4f) $A_{n+m}(\sigma)\left(Y_{n} \wedge Y_{m}\right)=A_{n}(\sigma) Y_{n} \wedge A_{m}(\sigma) Y_{m}$ for $Y_{n} \in D_{n}$ and $Y_{m} \in D_{m}$.

Proof. Choose $A_{0}$ as prescribed by (4e). Choose $A_{1}(\sigma)$ so that $A$ is $\sigma$-linear and such that $\partial_{1} A_{1}(\sigma)=A_{0}(\sigma) \partial_{1}$. Then define $A_{m}(\sigma)\left(X_{1} \wedge \cdots \wedge X_{m}\right)=A_{1}(\sigma) X_{1} \wedge \cdots$ $\wedge A_{1}(\sigma) X_{m}$.

Slightly less trivial is the following: Choose an ordering of the index set $J$.

Proposition. There is a collection of $U_{n}(\sigma, \tau)$ satisfying

(4g) $U_{0}(\sigma, \tau)=0$ and

(4h) $U_{n}(\sigma, \tau)\left(X_{i_{1}} \wedge \cdots \wedge X_{i_{n}}\right)=U_{n-1}(\sigma, \tau)\left(X_{i_{1}} \wedge \cdots \wedge X_{i_{n-1}}\right) \wedge A_{1}(\sigma) A_{1}(\tau) X_{i_{n}}$ $+(-1)^{n-1} A_{n-1}(\sigma, \tau)\left(X_{i_{1}} \wedge \cdots \wedge X_{i_{n-1}}\right) \wedge U_{1}(\sigma, \tau) X_{i_{n}}$ for $i_{1}<i_{2}<\cdots<i_{n}$. 
Proof. Choose $U_{0}$ as specified and let $U_{1}(\sigma, \tau)$ be any $\sigma \tau$-linear function satisfying $\partial_{2} U_{1}(\sigma, \tau)=A_{1}(\sigma, \tau)-A_{1}(\sigma) \cdot A_{1}(\tau)$. Define $U_{n}$ for $n \geqq 2$ by the formula above. We now inductively verify that $\partial_{n+1} U_{n}(\sigma, \tau)+U_{n-1}(\sigma, \tau) \partial_{n}=A_{n}(\sigma, \tau)$ $-A_{n}(\sigma) A_{n}(\tau)$.

For convenience we omit most of the complication in the subscripts.

$$
\begin{aligned}
\left(\partial_{n+1} U_{n}\right. & \left.+U_{n-1} \partial_{n}\right)\left(X_{1} \wedge \cdots \wedge X_{n}\right) \\
= & \partial_{n+1}\left((-1)^{n-1} A(\sigma, \tau)\left(X_{1} \wedge \cdots \wedge X_{n-1}\right) \wedge U_{1}\left(X_{n}\right)\right. \\
& \left.\quad+U_{n-1}\left(X_{1} \wedge \cdots \wedge X_{n-1}\right) A_{1}(\sigma) A_{1}(\tau)\left(X_{n}\right)\right) \\
& +U_{n-1}\left(\partial_{n-1}\left(X_{1} \wedge \cdots \wedge X_{n-1}\right) X_{n}+(-1)^{n-1}\left(X_{1} \wedge \cdots \wedge X_{n-1}\right) \partial_{1} X_{n}\right) \\
= & (-1)^{n-1} \partial_{n+1}\left(A(\sigma, \tau)\left(X_{1} \wedge \cdots \wedge X_{n-1}\right) \wedge U_{1}\left(X_{n}\right)\right) \\
& +\partial_{n} U_{n-1}\left(X_{1} \wedge \cdots \wedge X_{n-1}\right) A_{1}(\sigma)\left(A_{1}(\tau) X_{n}\right) \\
& +(-1)^{n} U_{n-1}\left(X_{1} \wedge \cdots \wedge X_{n-1}\right)\left(A_{0}(\sigma) A_{0}(\tau) \partial_{1} X_{n}\right) \\
& +(-1)^{n-2} A_{n-2}(\sigma, \tau) \partial_{n-1}\left(X_{1} \wedge \cdots \wedge X_{n-1}\right) U_{1} X_{n} \\
& +\left[U_{n-2} \partial_{n-1}\left(X_{1} \wedge \cdots \wedge X_{n-1}\right)\right] \\
& +\left(A_{1}(\sigma)\left(A_{1}(\tau) X_{n}\right)+(-1)^{n-1} U_{n-1}\left(X_{1} \wedge \cdots \wedge X_{n-1}\right) A_{0}(\sigma \tau) \partial_{1} X_{n}\right) .
\end{aligned}
$$

Here we have made use of the fact that the $\partial_{n-1}\left(X_{1} \wedge \cdots \wedge X_{n-1}\right) \wedge X_{n}$ is an alternating sum of $R$-basis elements for which our formula defining $U_{n-1}$ is defined and of the fact $U_{n-1}$ is $\sigma \tau$-linear.

Thus the expression becomes:

$$
\begin{aligned}
& (-1)^{n-1} \partial_{n-1}\left(A(\sigma, \tau)\left(X_{1} \wedge \cdots \wedge X_{n-1}\right) \wedge U_{1} X_{n}\right) \\
& +(\partial U+U \partial)\left(X_{1} \wedge \cdots \wedge X_{n-1}\right) \wedge A_{1}(\sigma)\left(A_{1}(\tau) X_{n}\right) \\
& +(-1)^{n-2} A_{n-2}(\sigma, \tau)\left(\partial_{n-1}\left(X_{1} \wedge \cdots \wedge X_{n-1}\right)\right) U_{1}\left(X_{n}\right) \\
& =(-1)^{n-1} A(\sigma, \tau) \partial_{n-1}\left(X_{1} \wedge \cdots \wedge X_{n-1}\right) \wedge U_{1} X_{n}+A_{n-1}(\sigma \tau)\left(X_{1} \wedge X_{n-1}\right) \\
& \cdot \partial_{2} U_{1} X_{n} \\
& +\left\{A_{n-1}(\sigma, \tau)-A_{n-1}(\sigma) A_{n-1}(\tau)\right\}\left(X_{1} \wedge \cdots \wedge X_{n-1}\right) \wedge A_{1}(\sigma)\left(A_{1}(\tau) X_{n}\right) \\
& +(-1)^{n-2} A_{n-2}(\sigma, \tau) \partial_{n-1}\left(X_{1} \wedge \cdots \wedge X_{n-1}\right) \wedge U_{1} X_{n} \\
& =A_{n-1}(\sigma, \tau)\left(X_{1} \wedge \cdots \wedge X_{n-1}\right)\left(A_{1}(\sigma, \tau) X_{n}-A_{1}(\sigma)\left(A_{1}(\tau) X_{n}\right)\right) \\
& +\left[A_{n-1}(\sigma, \tau)-A_{n-1}(\sigma) A_{n-1}(\tau)\right]\left(X_{1} \wedge \cdots \wedge X_{n-1}\right) A_{1}(\sigma)\left(A_{1}(\tau) X_{n}\right) \\
& =A_{n}(\sigma, \tau)\left(X_{1} \wedge \cdots \wedge X_{n}\right)-A_{n-1}(\sigma)\left(A_{n-1}(\tau)\left(X_{1} \wedge \cdots \wedge X_{n-1}\right)\right) \\
& \text { - } A_{1}(\sigma)\left(A_{1}(\tau) X_{n}\right) \\
& =A_{n}(\sigma, \tau)\left(X_{1} \wedge \cdots \wedge X_{n}\right)-A_{n}(\sigma)\left(A_{n-1}(\tau)\left(X_{1} \wedge \cdots \wedge X_{n-1}\right) \wedge A_{1}(\tau) X_{n}\right) \\
& =\left\{A_{n}(\sigma, \tau)-A_{n}(\sigma) \circ A_{n}(\tau)\right\}\left(X_{1} \wedge \cdots \wedge X_{n}\right) \text {. }
\end{aligned}
$$

We remark that the above proposition depends crucially on the ordering of the index set. I.e., the formulae defining $U_{n}$ do not hold for an arbitrary product $Y_{1} \wedge \cdots \wedge Y_{n}$ where $Y_{i} \in D_{1}$.

We use the following algebraic fact. 
Proposition 5. There are natural maps

$$
J_{n}: \operatorname{Hom}\left(\Lambda^{1} M, \Lambda^{2} M\right) \rightarrow \operatorname{Hom}\left(\Lambda^{n-1} M, \Lambda^{n} M\right)
$$

satisfying

$$
J_{n}(F)\left(x_{1} \wedge \cdots \wedge x_{n-1}\right)=\sum(-1)^{i+1} x_{1} \wedge \cdots \wedge x_{i-1} \wedge F\left(x_{1}\right) \wedge x_{i+1} \wedge \cdots \wedge x_{n-1}
$$

Proof. It is clear that a similar map

$$
J_{n}(\tilde{F}): \operatorname{Hom}\left(\Lambda^{1} M, \Lambda^{2} M\right) \rightarrow \operatorname{Hom}\left(\otimes^{n-1} M, \Lambda^{n} M\right)
$$

exists. We need only verify that $J_{n}(\tilde{F})$ annihilates elements of the form

$$
x_{1} \otimes \cdots \otimes x_{i} \otimes x \otimes x \otimes y_{1} \otimes \cdots \otimes y_{k}
$$

where $i+k+2=n-1$.

It is clear since $x \wedge x=0$ that all terms except perhaps two are separately zero and since $F(x) \in \Lambda^{2} M$ we have $x \wedge F(x)=F(x) \wedge x$.

Via canonical isomorphisms we may view $J_{n}$ as a homomorphism of $\Phi$-modules

$$
J_{n}: H^{1}\left(M, H_{2}(M)\right) \rightarrow H^{n-1}\left(M, H_{n}(M)\right) .
$$

THEOREM 6. $\left(J_{n+1}\right)_{*}\left(v^{2}(M)\right)=v^{n+1}(M)$.

Proof. Using the $\Phi$-system as above and expanding on - we see that

$$
\begin{aligned}
&-u^{n+1}(\sigma, \tau)\left(X_{i_{1}} \wedge \cdots \wedge X_{i_{n}}\right) \\
&=\sum(-1)^{j+1} X_{i_{1}} \wedge \cdots \wedge X_{i_{j}} \wedge\left(f^{2} U_{1}(\sigma, \tau) X_{i_{j+1}}\right) \wedge \cdots \wedge X_{n} .
\end{aligned}
$$

Untangling the definitions gives the theorem.

We have the remarkable corollaries.

Corollary. $v^{2}(M)=0$ iff $v^{n}(M)=0$ for all $n$.

Corollary. $2 v^{n}(M)=0$.

COROLlaRY. If $\Phi$ is a finite group with an odd number of elements, then $v^{n}(M)=0$ for all $n$.

This last corollary gives a much more satisfactory explanation of the facts alluded to in [2] concerning the integral representations of $Z_{p}$.

We remark, lest the reader become too optimistic, that $v^{n}(M) \neq 0$ in general. An example is given below.

Together with the results of [3], these corollaries give a fair hold on the 2nd differential in the Hochschild-Serre spectral sequence for any extension of a free abelian group.

2. The direct sum theorem. We suppose, in this section, that a $\Phi$-module $M=M^{\prime} \oplus M^{\prime \prime}$. We wish to express $v^{i}(M)$ in terms of $v^{j}\left(M^{\prime}\right)$ and $v^{n}\left(M^{\prime \prime}\right)$. In view of Theorem 6 of the preceding section the critical case is $v^{2}(M)$. A general formula follows from that special case. It turns out to be surprisingly complicated. 
Recalling the canonical isomorphisms

$$
\Lambda^{n}\left(M^{\prime} \oplus M^{\prime \prime}\right) \cong \bigoplus_{i+j=n} \Lambda^{i}\left(M^{\prime}\right) \otimes \Lambda^{j}\left(M^{\prime \prime}\right)
$$

we see that $H^{1}\left(M^{\prime}, H_{2}\left(M^{\prime}\right)\right) \cong \operatorname{Hom}\left(\Lambda^{1} M^{\prime}, \Lambda^{2} M^{\prime}\right)$ is a direct summand of $H^{1}\left(M, H_{2}\left(M^{\prime}\right)\right)$, as is $H^{1}\left(M, H_{2}(M)\right)$. In an obvious sense then we have the following theorem.

THEOREM 7. $v^{2}\left(M^{\prime} \oplus M^{\prime \prime}\right)=v^{2}\left(M^{\prime}\right)+v^{2}\left(M^{\prime \prime}\right)$.

Proof. Let $R^{\prime}=Z\left[M^{\prime}\right]$ and $R^{\prime \prime}=Z\left[M^{\prime \prime}\right]$; then $R=Z[M] \cong R^{\prime \prime} \otimes_{z} R^{\prime \prime}$. In fact $D_{*}=D_{*}^{\prime} \otimes D_{*}^{\prime \prime}$-for we may choose a basis for $M^{\prime} \oplus M^{\prime \prime}$ that is the union of a basis for $M^{\prime}$ and a basis for $M^{\prime \prime}$. It follows that we may find $A_{i}(\sigma)$ and $U_{i}(\sigma, \tau)$ for $D_{*}$ in terms of the corresponding homomorphisms in $D_{*}^{\prime}$ and $D_{*}^{\prime \prime}$. The details harbor no surprises and lead directly to the formula given.

For the convenience of the reader we formulate precisely the more general formula. For this purpose we introduce the following notation:

$$
\begin{aligned}
C_{j}^{\prime}: \operatorname{Hom}\left(\Lambda^{i} M^{\prime}, \Lambda^{i+1} M^{\prime}\right) & \rightarrow \operatorname{Hom}\left(\Lambda^{i+j} M, \Lambda^{i+j+1} M\right) \\
C_{j}^{\prime}(F)(x \otimes y)=F(x) \otimes y & \text { if } x \in \Lambda^{i} M^{\prime} \text { and } y \in \Lambda^{j} M^{\prime \prime} \\
=0 & \text { otherwise. }
\end{aligned}
$$

Of course we are making use of the canonical isomorphisms above.

Similarly we have

$$
\begin{array}{rlrl}
C_{j}^{\prime \prime}: \text { Hom }\left(\Lambda^{i} M^{\prime \prime}, \Lambda^{i+1} M^{\prime \prime}\right) \rightarrow & \operatorname{Hom}\left(\Lambda^{i+j} M, \Lambda^{i+j+1} M\right) \\
C_{j}^{\prime \prime}(F)(x \otimes y) & =(-1)^{j} x \otimes F(y) & & \text { if } x \in \Lambda^{j} M^{\prime} \text { and } y \in \Lambda^{i} M^{\prime \prime} \\
& =0 & & \text { otherwise. }
\end{array}
$$

With this notation then we have the following formula.

THEOREM 8. $v^{n+1}\left(M^{\prime} \oplus M^{\prime \prime}\right)=\sum_{i+j=n} C_{j^{\star}}^{\prime}\left(v^{i+1}\left(M^{\prime}\right)\right)+C_{i^{*}}^{\prime \prime}\left(v^{j+1}\left(M^{\prime \prime}\right)\right)$.

The proof is immediate from Theorem 6 and the previous theorem.

ExAMPLE 3. $\Phi=Z_{2}+Z_{2}, M=$ the group ring of $\Phi$ modulo its invariant elements, i.e.

$$
0 \longrightarrow Z \stackrel{j}{\longrightarrow} Z[\Phi] \stackrel{p}{\longrightarrow} M \longrightarrow 0
$$

is an exact sequence of $\Phi$-modules with $j(1)=\sum_{\sigma \in \Phi} \sigma$.

Let $\varepsilon_{1}$ and $\varepsilon_{2}$ be generators of $\Phi$ and define $m_{i}=\rho\left(\varepsilon_{i}\right) i=1,2$ and $m_{3}=\rho\left(\varepsilon_{1} \varepsilon_{2}\right)$. It is easy to see that relative to the basis $\left(m_{1}, m_{2}, m_{3}\right) \varepsilon_{1}$ and $\varepsilon_{2}$ induce the transformations described by the matrices

$$
\left(\begin{array}{lll}
-1 & 0 & 0 \\
-1 & 0 & 1 \\
-1 & 1 & 0
\end{array}\right) \text { and }\left(\begin{array}{lll}
0 & -1 & 1 \\
0 & -1 & 0 \\
1 & -1 & 0
\end{array}\right)
$$


Let $t_{i}=m_{i}-1 \in R=Z[M]$. It is easy to see that

$$
\begin{array}{ll}
\varepsilon_{1}\left(t_{1}\right)=-\left\{m_{1}^{-1} m_{3}^{-1} t_{1}+m_{1}^{-1} m_{2}^{-1} m_{3}^{-1} t_{2}+m_{3}^{-1} t_{3}\right\} \\
\varepsilon_{1}\left(t_{2}\right)= & t_{3} \\
\varepsilon_{1}\left(t_{3}\right)= & t_{2}
\end{array}
$$

and hence that one may define $A_{1}\left(\varepsilon_{1}\right)$ by

$$
\begin{aligned}
& A_{1}\left(\varepsilon_{1}\right): X_{1} \mapsto-\left\{m_{1}^{-1} m_{3}^{-1} X_{1}+m_{1}^{-1} m_{2}^{-1} m_{3}^{-1} X_{2}+m_{3}^{-1} X_{3}\right\} \\
& X_{2} \mapsto \quad X_{3} \\
& X_{3} \mapsto \quad X_{2}
\end{aligned}
$$

Similarly one can show that the following is a permissible choice for $A_{1}\left(\varepsilon_{2}\right)$.

$$
\begin{array}{rlr}
A_{1}\left(\varepsilon_{2}\right): X_{1} & \mapsto & X_{3} \\
X_{2} & \mapsto & -\left\{m_{1}^{-1} m_{2}^{-1} m_{3}^{-1} X_{1}+m_{2}^{-1} m_{3}^{-1} X_{2}+m_{3}^{-1} X_{3}\right\} \\
X_{3} & \mapsto & X_{1}
\end{array}
$$

A direct computation shows that both $A_{1}\left(\varepsilon_{1}\right) \circ A_{1}\left(\varepsilon_{1}\right)$ and $A_{1}\left(\varepsilon_{2}\right) \circ A_{1}\left(\varepsilon_{2}\right)=$ the identity map: $D_{1} \rightarrow D_{1}$. (Recall that $A_{1}\left(\varepsilon_{i}\right)$ is $\varepsilon_{i}$-linear!)

We may choose $A_{1}\left(\varepsilon_{1} \varepsilon_{2}\right)=A_{1}\left(\varepsilon_{1}\right) \circ A_{1}\left(\varepsilon_{2}\right)$ and $A_{1}(\mathrm{id})=$ identity. This completes the computation of $A_{*}$ in view of Proposition 4. It is now, of course, possible to compute all of $U_{i}(\sigma, \tau)$, but not all of them need be computed. Formula (7) gives an explicit nonhomogeneous two-dimensional $\Phi$-cocycle with coefficients in $H^{1}\left(M, H_{2}(M)\right)$ which represents $v^{2}(M)$. In view of the special choice of $\Phi$ it is possible to describe a two-dimensional cocycle much more efficiently.

Letting $\Omega=Z[\Phi]$ we may construct a $\Omega$-free acyclic resolution, $T_{*}$ of $Z$ as follows :

$$
\ldots \stackrel{\partial_{3}}{\longrightarrow} \Omega \alpha_{0,2} \oplus \Omega \alpha_{1,1} \oplus \Omega \alpha_{2,0} \stackrel{\partial_{2}}{\longrightarrow} \Omega \alpha_{0,1} \oplus \Omega \alpha_{1,0} \stackrel{\partial_{1}}{\longrightarrow} \Omega \alpha_{0,0} \stackrel{\varepsilon}{\longrightarrow} Z \longrightarrow 0 .
$$

Here $\alpha_{i, j}$ is a free generator of $T_{i+j}$. The maps in question are

$$
\begin{aligned}
\varepsilon\left(\alpha_{0,0}\right) & =1, \\
\partial_{1}: \alpha_{1,0} & \mapsto\left(\varepsilon_{1}-1\right) \alpha_{0,0}, \\
\alpha_{0,1} & \mapsto\left(\varepsilon_{2}-1\right) \alpha_{0,0}, \\
\partial_{2}: \alpha_{2,0} & \mapsto\left(\varepsilon_{1}+1\right) \alpha_{1,0}, \\
\alpha_{1,1} & \mapsto\left(\varepsilon_{1}-1\right) \alpha_{0,1}-\left(\varepsilon_{2}-1\right) \alpha_{1,0}, \\
\alpha_{0,2} & \mapsto\left(\varepsilon_{2}+1\right) \alpha_{0,1} .
\end{aligned}
$$


A chain map $\psi_{*}$ from this resolution to the standard nonhomogeneous resolution (see [1] for terminology) is given by

$$
\begin{aligned}
\psi_{0}: \alpha_{0,0} & \mapsto[], \\
\psi_{1}: \alpha_{1,0} & \mapsto\left[\varepsilon_{1}\right], \\
\alpha_{0,1} & \mapsto\left[\varepsilon_{2}\right], \\
\psi_{2}: \alpha_{2,0} & \mapsto\left[\varepsilon_{1}, \varepsilon_{1}\right]+[1,1], \\
\alpha_{1,1} & \mapsto\left[\varepsilon_{1}, \varepsilon_{2}\right]-\left[\varepsilon_{2}, \varepsilon_{1}\right], \\
\alpha_{0,2} & \mapsto\left[\varepsilon_{2}, \varepsilon_{2}\right]+[1,1] .
\end{aligned}
$$

It follows that if $h$ is any nonhomogeneous 2-cocycle with coefficients in the $\Phi$-module $A$, then $h \circ \psi_{2}$ represents the same cohomology class. Then formula (7) shows that $v^{2}(M) \in H^{2}\left(\Phi, H^{1}\left(M, H_{2}(M)\right)\right.$ is determined by $\omega^{2}$ where

$$
\begin{aligned}
& \omega^{2} \circ \psi_{2}\left(\alpha_{2,0}\right)=-\left\{\varepsilon_{1}^{-1} \varepsilon_{1}^{-1} f_{2} U_{1}\left(\varepsilon_{1}, \varepsilon_{1}\right)+1 \cdot 1 \cdot f_{2} U_{1}(1,1)\right\} \in \operatorname{Hom}\left(H_{1}, H_{2}\right), \\
& \omega^{2} \circ \psi_{2}\left(\alpha_{1,1}\right)=-\left\{\varepsilon_{1}^{-1} \varepsilon_{2}^{-1} f_{2} U_{1}\left(\varepsilon_{1}, \varepsilon_{2}\right)-\varepsilon_{2}^{-1} \varepsilon_{1}^{-1} f_{2} U_{1}\left(\varepsilon_{2}, \varepsilon_{1}\right)\right\},
\end{aligned}
$$

and

$$
\omega^{2} \circ \psi_{2}\left(\alpha_{0,2}\right)=-\left\{\varepsilon_{2}^{-1} \varepsilon_{2}^{-1} f_{2} U_{1}\left(\varepsilon_{2}, \varepsilon_{2}\right)+1 \cdot 1 \cdot f_{2} U_{1}(1,1)\right\} .
$$

In view of the remarks above that $A_{1}\left(\varepsilon_{i}\right)^{2}=$ id we may choose $U_{1}\left(\varepsilon_{i}, \varepsilon_{i}\right)=0$. As usual we may choose $U_{1}(1,1)=0$. Since $A_{1}\left(\varepsilon_{1} \varepsilon_{2}\right)=A_{1}\left(\varepsilon_{1}\right) A_{1}\left(\varepsilon_{2}\right)$ by definition we may choose $U_{1}\left(\varepsilon_{1}, \varepsilon_{2}\right)=0$. Thus our cocycle takes the form

$$
\begin{aligned}
& \alpha_{2,0} \mapsto 0, \\
& \alpha_{1,1} \mapsto\left\{\varepsilon_{2}^{-1} \varepsilon_{1}^{-1} f_{2} U_{1}\left(\varepsilon_{2}, \varepsilon_{1}\right)\right\}, \\
& \alpha_{0,2} \mapsto 0 .
\end{aligned}
$$

We must now calculate $U_{1}\left(\varepsilon_{2}, \varepsilon_{1}\right)$. We have

$$
\begin{aligned}
\partial_{2} U_{1}\left(\varepsilon_{2}, \varepsilon_{1}\right) & =A_{1}\left(\varepsilon_{2} \varepsilon_{1}\right)-A_{1}\left(\varepsilon_{2}\right) \circ A_{1}\left(\varepsilon_{1}\right) \\
& =A_{1}\left(\varepsilon_{1}\right) \circ A_{1}\left(\varepsilon_{2}\right)-A_{1}\left(\varepsilon_{2}\right) \circ A_{1}\left(\varepsilon_{1}\right) .
\end{aligned}
$$

A direct calculation shows that this right-hand side is the homomorphism

$$
\begin{aligned}
& X_{1} \mapsto\left(m_{1}^{-1}-m_{1}^{-1} m_{3}^{-1}\right) X_{1}+\left(1-m_{3}^{-1}\right) X_{2}+\left(m_{1}^{-1} m_{3}^{-1}-m_{2} m_{3}^{-1}\right) X_{3}, \\
& X_{2} \mapsto\left(m_{3}^{-1}-1\right) X_{1}+\left(m_{2}^{-1} m_{3}^{-1}-m_{2}^{-1}\right) X_{2}+\left(m_{1} m_{3}^{-1}-m_{2}^{-1} m_{3}^{-1}\right) X_{3}, \\
& X_{3} \mapsto\left(m_{1}^{-1} m_{2}^{-1} m_{3}^{-1}-m_{1}^{-1} m_{3}^{-1}\right) X_{1}+\left(m_{2}^{-1} m_{3}^{-1}-m_{1}^{-1} m_{2}^{-1} m_{3}^{-1}\right) X_{2}+0,
\end{aligned}
$$

and thus one can choose $U_{1}\left(\varepsilon_{2}, \varepsilon_{1}\right)$ to be

$$
\begin{aligned}
U_{1}\left(\varepsilon_{2}, \varepsilon_{1}\right): X_{1} & \mapsto 0-m_{1}^{-1} m_{3}^{-1} X_{1} \wedge X_{3}+m_{3}^{-1} X_{2} \wedge X_{3}, \\
X_{2} & \mapsto 0-m_{3}^{-1} X_{1} \wedge X_{3}-m_{2}^{-1} m_{3}^{-1} X_{2} \wedge X_{3}, \\
X_{3} & \mapsto-m_{1}^{-1} m_{2}^{-1} m_{3}^{-1} X_{1} \wedge X_{2}+0+0 .
\end{aligned}
$$


Consequently if we identify $H_{i}(M)$ with $\Lambda^{i} M$

$$
\begin{aligned}
\phi=\varepsilon_{2}^{-1} \varepsilon_{1}^{-1} f_{2} U_{1}\left(\varepsilon_{2}, \varepsilon_{1}\right): m_{1} & \mapsto 0+m_{1} \wedge m_{3}+m_{2} \wedge m_{3}, \\
m_{2} & \mapsto 0-m_{1} \wedge m_{3}-m_{2} \wedge m_{3}, \\
m_{3} & \mapsto-m_{1} \wedge m_{3} .
\end{aligned}
$$

Summarizing. $v^{2}(M) \in H^{2}\left(\Phi ; \operatorname{Hom}\left(\Lambda^{1} M, \Lambda^{2} M\right)\right)$ is given by the cocycle sending $\alpha_{2,0}$ and $\alpha_{0,2}$ to zero but sending $\alpha_{1,1}$ to the homomorphism, $\phi$, just described.

We now contend that $v^{2}(M) \neq 0$. For suppose $g$ is a 1 -cochain whose coboundary is the above cocycle. Then if

$$
y_{0,1}=g\left(\alpha_{0,1}\right) \in \operatorname{Hom}\left(M, \Lambda^{2}(M)\right), \text { and } y_{1,0}=g\left(\alpha_{1,0}\right),
$$

then we would have

(1) $0=\left(1+\varepsilon_{1}\right) y_{0,1}$,

(2) $\phi=\left(\varepsilon_{1}-1\right) y_{0,1}-\left(\varepsilon_{2}-1\right) y_{1,0}$, and

(3) $0=\left(1+\varepsilon_{2}\right) y_{1,0}$.

We now make use of the structure of $M$ as a module over the subgroups generated by $\varepsilon_{i}$. Equation (1) implies that $y_{0,1}$ defines an element in $H^{1}\left(Z_{2}^{(1)}, \operatorname{Hom}\left(M, \Lambda^{2} M\right)\right)$ where $Z_{2}^{(1)}=$ the subgroup of $\Phi$ generated by $\varepsilon_{1}$. A similar statement holds for $y_{1,0}$. A lemma proved below shows $H^{1}\left(Z_{2}^{(i)}, \operatorname{Hom}\left(M, \Lambda^{2} M\right)\right)=0$. Thus we conclude the existence of $x_{0,1}$ and $x_{1,0} \in \operatorname{Hom}\left(M, \Lambda^{2} M\right)$ such that $y_{1,0}=\left(\varepsilon_{1}-1\right) x_{1,0}$ and $y_{0,1}=\left(\varepsilon_{2}-1\right) x_{0,1}$. Thus equation (2) implies the existence of $\xi$ such that

$$
\phi=\left(\varepsilon_{1}-1\right)\left(\varepsilon_{2}-1\right) \cdot \xi \in \operatorname{Hom}\left(M, \Lambda^{2} M\right) .
$$

We now demonstrate that such an equation is impossible. Note that $M=\Lambda^{1} M$ and $\Lambda^{2} M$ are paired to $\Lambda^{3} M$ which is easily seen to be a trivial $\Phi$-module of rank 1 with $m_{1} \wedge m_{2} \wedge m_{3}$ as generator.

Note that

$\phi\left(m_{2}\right) \wedge m_{3}-\phi\left(m_{1}\right) \wedge m_{2}=\left(-m_{1} \wedge m_{3}-m_{2} \wedge m_{3}\right) \wedge m_{3}$

$$
-\left(m_{1} \wedge m_{3}+m_{2} \wedge m_{3}\right) \wedge m_{2}=0+m_{1} \wedge m_{2} \wedge m_{3}
$$

is a generator. On the other hand

$$
\begin{aligned}
\left\{\left(\varepsilon_{1}-1\right)\left(\varepsilon_{2}-1\right) \xi\right\}\left(m_{2}\right) \wedge m_{3}-\left\{\left(\varepsilon_{1}-1\right)\left(\varepsilon_{2}-1\right) \xi\right\} & \left(m_{1}\right) \wedge m_{2} \\
& =-2 \xi\left(m_{1}+m_{3}\right) \wedge\left(m_{2}+m_{3}\right)
\end{aligned}
$$

(detail is easy though tedious) which cannot be a generator.

Lemma. $H^{1}\left(Z_{2}^{(i)}, \operatorname{Hom}\left(M, \Lambda^{2} M\right)\right)=0$.

Proof. As we have already remarked, $\Lambda^{3} M \simeq Z$ is a trivial $\Phi$-module and hence $\Lambda^{2} M \simeq \operatorname{Hom}_{Z}(M, Z) \equiv M^{*}$. We have the exact sequence

$$
0 \rightarrow Z \rightarrow Z[\Phi] \rightarrow M \rightarrow 0 \text {. }
$$

Thus

$$
0 \leftarrow M^{*} \leftarrow \operatorname{Hom}\left(Z[\Phi], M^{*}\right) \leftarrow \operatorname{Hom}\left(M, M^{*}\right) \leftarrow 0
$$


According to [1, p. 199], the middle module is $Z[\Phi]$-free and hence $H^{j}\left(Z_{2}^{(i)}, \operatorname{Hom}\left(M, M^{*}\right)\right) \simeq H^{j-1}\left(Z_{2}^{(i)}, M^{*}\right)$ for $j>1$; and from

$$
0 \rightarrow M^{*}=Z[\Phi]^{*} \rightarrow Z^{*} \rightarrow 0
$$

we conclude that $H^{j-1}\left(Z_{2}^{(i)}, M^{*}\right) \simeq H^{j-1}\left(Z_{2}^{(i)}, Z^{*}\right)=$ for $j-1>1$. But it is well known that $H^{j}\left(Z_{2}^{(i)}, Z\right)$ is zero for odd $i$ and $H^{j}\left(Z_{2}, \quad\right)$ is periodic of order 2. This completes the proof that $v^{2}(M) \neq 0$.

The methods of the previous lemma can be used to determine the group in which $v^{2}$ lies, i.e. $H^{2}\left(\Phi, H^{\prime}\left(M, H_{2}(M)\right)\right)$. Since $(*),\left(*^{\prime}\right),\left(*^{\prime \prime}\right)$ are exact sequences of $\Phi$-modules, we get

$$
\begin{aligned}
H^{2}\left(\Phi, H^{1}\left(M, H_{2}(M)\right)\right) & \simeq H^{2}\left(\Phi, \operatorname{Hom}\left(M, \Lambda^{2} M\right)\right) \\
& \simeq H^{2}\left(\Phi, \operatorname{Hom}\left(M, M^{*}\right)\right) \simeq H^{1}\left(\Phi, M^{*}\right) .
\end{aligned}
$$

But $Z[\Phi]^{*} \simeq Z[\Phi]$, so $\left(*^{\prime \prime}\right)$ shows that $M^{*} \simeq I$, the augmentation ideal of $Z[\Phi]$. In any case we have from $\left(* *^{\prime \prime}\right)$

$$
\cdots \longrightarrow H^{0}(\Phi, Z[\Phi]) \stackrel{\varepsilon_{*}}{\longrightarrow} H^{0}(\Phi, Z) \longrightarrow H^{1}\left(\Phi, M^{*}\right) \longrightarrow 0
$$

and $H^{\circ}(\Phi, Z[\Phi])=$ ideal generated by $\sum=\sum_{\sigma \in \Phi}$ in $Z[\Phi]$ and $H^{\circ}(\Phi, Z)=Z$, so the question is, what is $\varepsilon\left(\sum\right)$ ? But $\varepsilon$ is just the usual augmentation map, so $\varepsilon\left(\sum\right)=4$ and $H^{1}\left(\Phi, M^{*}\right)=Z_{4}$, so we have proved

THEOREM 9. Let $\Phi$ be $Z_{2} \oplus Z_{2} \& M$ the quotient of $Z[\Phi]$ by the ideal generated by $\sum=\sum_{\sigma \in \Phi} \sigma$. Then

$$
H^{2}\left(\Phi, H^{1}\left(M, H_{2}(M)\right)\right)=Z_{4}
$$

and $v^{2}(M)$ is the element of order 2 in $Z_{4}$.

Note that $v^{i}(M)=0$ for $i>2$, since $v^{i}(M)=0$ for $i>3$ since $M$ has rank 3 and $v^{3}$ happens to lie in a 0 group, i.e.

$$
\begin{aligned}
H^{2}\left(\Phi, H^{2}\left(M, H_{3}(M)\right)\right)= & H^{2}\left(\Phi, H^{2}(M)\right)=H^{2}\left(\Phi, \Lambda^{2} M\right) \\
= & H^{2}\left(\Phi, M^{*}\right)=H^{1}(\Phi, Z)=\left[H^{0}\left(Z_{2}, Z\right) \otimes H^{1}\left(Z_{2}, Z\right)\right] \\
& \otimes\left[H^{1}\left(Z_{2}, Z\right) \otimes H^{0}\left(Z_{2}, Z\right)\right]=0 .
\end{aligned}
$$

REMARK. The above is the only example we know of a nonzero characteristic class of a $Z$-free module.

EXAMPLE 2*. In this example we briefly consider the dual of

$$
M, M^{*}=\operatorname{Hom}(M, Z)=I,
$$

the augmentation ideal of $Z[\Phi]$. Although $M$ and $I$ are closely related, we prove

Proposition A. $H^{2}\left(\Phi, H^{1}\left(I, H_{2}(I)\right)\right)=Z_{2} \oplus Z_{2} \oplus Z_{2}$.

Proposition B. $v^{i}(I)=0$ for all $i$.

For Proposition A, we merely list the string of isomorphisms:

$$
\begin{aligned}
H^{2}\left(\Phi, H^{1}\left(I, H_{2}(I)\right)\right) & =H^{2}\left(\Phi, \operatorname{Hom}\left(I, \Lambda_{2} I\right)\right)=H^{2}(\Phi, \operatorname{Hom}(I, M)) \\
& =H^{3}(\Phi, M)=H^{4}(\Phi, Z)=Z_{2} \oplus Z_{2} \oplus Z_{2} .
\end{aligned}
$$


For B, we list a $\Phi$-system for the associated Koszul complex which has the property that $A_{1}(\lambda) \circ A_{1}(\eta)=A_{1}(\eta) \circ A_{1}(\lambda)$ and $\left[A_{1}(\sigma)\right]^{2}=\left[A_{1}(\tau)\right]^{2}=\left[A_{1}(\sigma \tau)\right]^{2}=$ the identity. Hence we get $U \equiv 0$, so by Theorem $5, U_{*} \equiv 0$. Let $i_{1}, i_{2}, i_{3}$ be the generators of $I$, and

$$
\begin{aligned}
& \sigma \cdot i_{1}=i_{1}^{-1} \\
& \tau \cdot i_{1}=i_{3} i_{2}^{-1} \\
& \sigma \cdot i_{2}=i_{3} i_{1}^{-1} \\
& \tau \cdot i_{2}=i_{2}^{2} \\
& \sigma \cdot i_{3}=i_{2} i_{1}^{-1} \\
& \tau \cdot i_{3}=i_{1} i_{2}^{-1} \\
& {\left[A_{1}(\sigma)\right]\left(X_{1}\right)=-i_{1}^{-1} X_{1}} \\
& {\left[A_{1}(\tau)\right]\left(X_{1}\right)=-i_{3} i_{2}^{-1} X_{2}+X_{3}} \\
& {\left[A_{1}(\sigma)\right]\left(X_{2}\right)=-i_{3} i_{1}^{-1} X_{1}+X_{3}} \\
& {\left[A_{1}(\tau)\right]\left(X_{2}\right)=-i_{2}^{-1} X_{2}} \\
& {\left[A_{1}(\sigma)\right]\left(X_{3}\right)=-i_{2} i_{1}^{-1} X_{1}+X_{2}} \\
& {[A(\tau)]\left(X_{3}\right)=-i_{1} i_{2}^{-1} X_{2}+X_{1} \text {. }}
\end{aligned}
$$

3. Future developments. First, we would like to say that it now seems likely that the main results of [3] can be reformulated more generally so as to omit all restrictions on the final coefficients $\Gamma$ and to make more obvious the applications to other categories, e.g. Lie algebras, associative algebras, etc. If this turns out to be the case, the general theory of this paper will then apply to this less restrictive situation.

As for the category of modules over groups, in a later paper we hope to investigate the case in which the module is finite in a similar manner that the $Z$-free case was examined in the second half of this paper. Eventually there should be a unified treatment which would presumably utilize a complex defined by Tate.

Finally, the theorems of $\S 1$ show that $v^{2}(M)$ is really the object of primary concern (at least in the $Z$-free case). We would like to find classes

$$
v^{(i)} \in H^{i}\left(\Phi, H^{i-1}\left(M, H_{i}(M)\right)\right)
$$

with $v^{(2)}=v^{2}$ and with $v^{(i)}$ determining the differential $d_{i}$ in the spectral sequence for the split extension $\Phi \cdot M$. Note that if one takes final coefficients $\Gamma=Z_{3}$, say, then we know that $d_{2}=0$, and it is easy to see that $d_{3}$ on the third row is given by a cup product with an element

$$
v^{(3)} \in H^{3}\left(\Phi, H^{2}\left(M, H_{3}\left(M, Z_{3}\right)\right)\right) .
$$

\section{BIBLIOGRAPHY}

1. H. Cartan and S. Eilenberg, Homological algebra, Princeton Univ. Press, Princeton, N. J., 1956.

2. L. S. Charlap and A. T. Vasquez, The cohomology of group extensions, Bull. Amer. Math. Soc. 69 (1963), 815-817.

3. - The cohomology of group extensions, Trans. Amer. Math. Soc. 124 (1966), 24-40.

4. G. Hochschild and J.-P. Serre, The cohomology of group extensions, Trans. Amer. Math. Soc. 74 (1953), 110-134.

University of Pennsylvania, Philadelphia, Pennsylvania

BRANDEIS UNIVERSITY, Waltham, Massachusetts 